\title{
Evaluation of Short-Term Pacing Effect to Predict Long-Term Response to Cardiac Resynchronization Therapy: the TRAJECTORIES Study
}

\author{
Giulia Santarelli ${ }^{1}$, Roberta Ciccotelli ${ }^{1}$, Giulio Molon ${ }^{2}$, Francesco Zanon ${ }^{3}$, Alessandro Corzani ${ }^{4}$, \\ Antonio Rossillo ${ }^{5}$, Mauro Biffi ${ }^{6}$, Gabriele Zanotto ${ }^{7}$, Laura Lanzoni ${ }^{2}$, Stefano Severi ${ }^{1}$, Corrado \\ Tomasi $^{8}$, Cristiana Corsi ${ }^{1}$ \\ ${ }^{1}$ DEI, University of Bologna, Cesena, Italy \\ ${ }^{2}$ Cardiology Dept, IRCCS Sacro Cuore Don Calabria Hospital, Negrar, Verona, Italy \\ ${ }^{3}$ Santa Maria della Misericordia, Rovigo, Italy \\ ${ }^{4}$ Bufalini Hospital, AUSL della Romagna, Cesena, Italy \\ ${ }^{5}$ San Bortolo Hospital, ULSS 8 Berica, Vicenza, Italy \\ ${ }^{6}$ Sant'Orsola-Malpighi University Hospital, Bologna, Italy \\ ${ }^{7}$ Mater Salutis Hospital, Legnago, Verona, Italy \\ ${ }^{8}$ Santa Maria delle Croci Hospital, AUSL della Romagna, Ravenna, Italy
}

\begin{abstract}
Cardiac resynchronization therapy (CRT) is an effective treatment for chronic symptomatic systolic heart failure with cardiac dyssynchrony, but about 1/3 of patients do not respond favorably to the therapy. We hypothesized that acute modifications of the coronary sinus (CS) pacing cathode movements induced by biventricular pacing may be related to resynchronization process and consequently may carry predictive power on $C R T$ response. A method for the $3 D$ reconstruction of $C S$ lead's pacing cathode trajectory (3DTJ) throughout a cardiac cycle showed that trajectory's geometry suddenly changed in responders $(R)$ upon starting of biventricular pacing, becoming less eccentric and more multidirectional. Our multicenter observational study aimed at evaluating the clinical value of 3DTJ. Out of 119 patients enrolled, 50 have ended follow-up and have been analyzed. Concordance between 3DTJ metrics and response was $82 \%$ overall (41/50), 91\% in R (31/34), 62\% in NR (10/16). The proposed 3DTJ metric showed high sensitivity (91\%) with specificity $=62 \% ; P P V=84 \%, N P V=77 \%$. From our data, 3DTJ seems a promising tool to acutely predict CS pacing site-specific response to CRT. Its investigational use as an intra-operatory, real-time guidance for selecting $L V$ pacing sites may open new opportunities for CRT patients' selection and therapy delivery.
\end{abstract}

\section{Introduction}

In the large population of patients with drug-refractory heart failure, chronic symptomatic systolic heart failure and cardiac dyssynchrony, cardiac resynchronization therapy (CRT) has shown impressive results [1]. Several studies demonstrated that CRT lowers mortality [2] and improves both exercise tolerance and quality of life [3]. These benefits depend on LV "reverse remodeling", which consists of an improvement of systolic function and of a reduction of left ventricular (LV) volumes [4]. However, about one-third of patients do not respond to CRTs [3,5] and the reasons of failure are still unclear and may be related to various factors, among which some regarding lead-vein-myocardium interactions, such as a suboptimal or non-continuous stimulation, an unsuitable or unstable location in the coronary sinus (CS) branch, and an illdefined incapacity to change myocardial mechanics.

In our previous works [6,7], the CS pacing lead tip movements were investigated as a source of information about acute rearrangement of $\mathrm{LV}$ mechanics in CRT recipients. Results in 22 patients from a single center showed that trajectory's geometry at biventricular pacing (BIV) start, suddenly changed only in responder patients, becoming less eccentric and more multi-directional, as described by the ratio between its two first singular values.

In this study, we evaluated the clinical value of these observations in a large cohort of CRT patients in a multicenter study. We investigated and validated the hypothesis that acute modifications of the CS lead pacing cathode trajectory induced by CRT may offer clues about the underlying resynchronization process and the efficacy of the procedure.

Therefore, studying the CS lead trajectory before and immediately after the turning-on of biventricular pacing at the implant could make it possible to derive prognostic information about long-term responses to CRT. 
A. fluoroscopic data acquisition

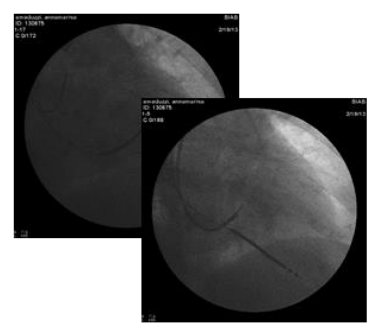

B. pacing cathode tracking and segmentation

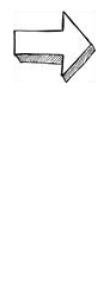

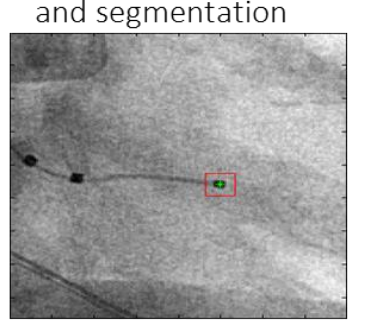

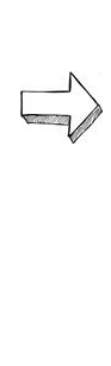

E. trajectory computations
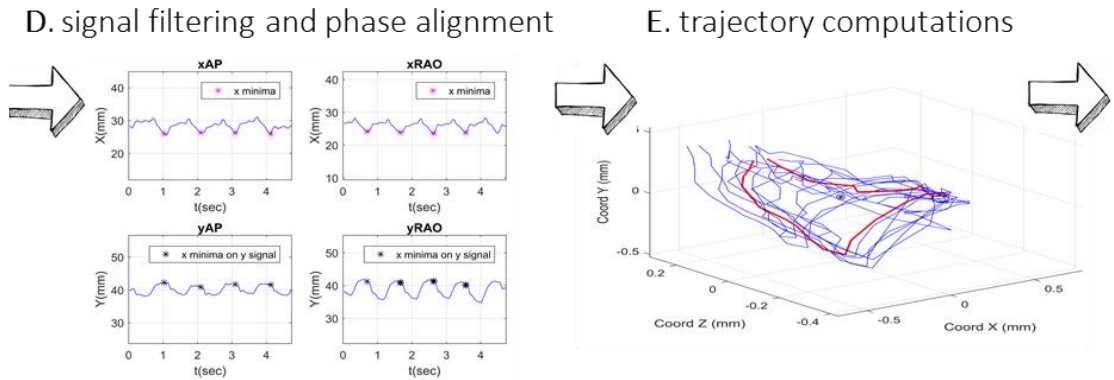

C. coordinates of the pacing cathode

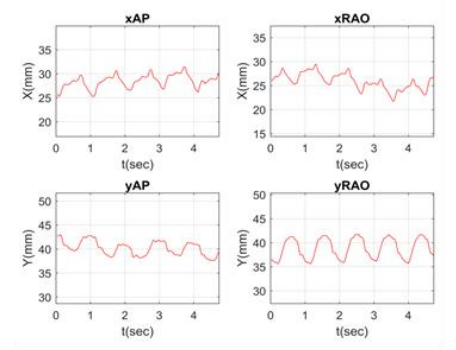

F. final «average» trajectory and parameter computation

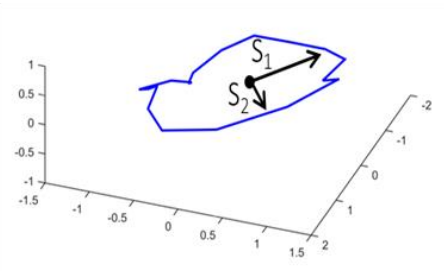

Fig. 1: Schematic of fluoroscopic data analysis workflow to extract parameters describing shape trajectory changes due to biventricular pacing start (see text for details).

\section{Materials and Methods}

\subsection{Clinical Data}

In seven hospitals in the Emilia-Romagna and Veneto regions, in Italy, 119 patients undergoing CRT because of conventional class I indication, were studied after obtaining their informed consent (Trajectory Changes of Coronary Sinus Lead Tip and Cardiac Resynchronization Therapy Outcome, NCT02340546).

Out of 119 patients enrolled in 42 months, 50 patients have ended follow-up ( $35 \mathrm{M}$; age $69 \pm 11 \mathrm{y}$ ) and have been analysed, while 10 dropped-out. Fluoroscopies were acquired in the three standard views (AP: antero-posterior; RAO: right anterior oblique; LAO: left anterior oblique).

Patients baseline features were: ischemic heart disease (IHD) in 28/50 patients; sinus rhythm in $43 / 50$ patients; upgrade from pacemaker/implantable defibrillator device in 9/50 patients; QRS morphology with left bundle branch block (LBBB) in 40/50 patients; intraventricular aspecific delay in 3 and right ventricular (RV) pace in 8 patients; $\mathrm{LV}$ ejection fraction (LVEF) was $32 \pm 10 \%$; QRS duration was $162 \pm 26 \mathrm{msec}$.

\subsection{Data Processing}

In our previous works [6,7], to evaluate the CS lead dynamic position and movements we processed the $\mathrm{x}$-ray fluoroscopy sequences acquired immediately before $\left(t_{-1}\right)$ and just after $\left(\mathrm{t}_{0}\right)$ the turning-on of biventricular pacing. An automated method for 3D reconstruction of CS lead's pacing cathode trajectory (3DTJ) throughout a cardiac cycle was designed and implemented.

The workflow is depicted in Figure 1. Briefly, we designed a tracking procedure based on a fast region matching approach to detect and follow the CS lead cathode pole throughout the cardiac cycle from two standard fluoroscopic views (Figure1A and 1B). This step results in the coordinates of the pole in the two analyzed views (Figure 1C). These signals were then filtered to compensate for motion due to respiration and aligned (Figure 1D); by applying stereo-photogrammetric rules, from the coordinates in both views, the $3 \mathrm{D}$ trajectories over several consecutive cardiac cycles were reconstructed (Figure 1E) and the mean trajectory over one cycle was then considered for the extraction of several parameters, including the ratio of the two main singular values $\left(\mathrm{S}_{1} / \mathrm{S}_{2}\right)$ of the matrix containing the trajectory coordinates. This analysis was repeated using fluoroscopic data acquired at $t_{1}$ and $t_{0}$ and the change in $S_{1} / S_{2}\left(\Delta S_{1} / S_{2}\right)$ was also computed.

$\Delta \mathrm{S}_{1} / \mathrm{S}_{2}$ between pre-CRT and CRT start was compared with the volumetric response to the therapy at six-month follow-up: the percent decrease of $S_{1} / S_{2}$ induced by BIV, marking a more multi-directional shape of 3DTJ, was assumed to predict the response to CRT. Representative examples of the trajectory shapes are shown in Figure 2. 

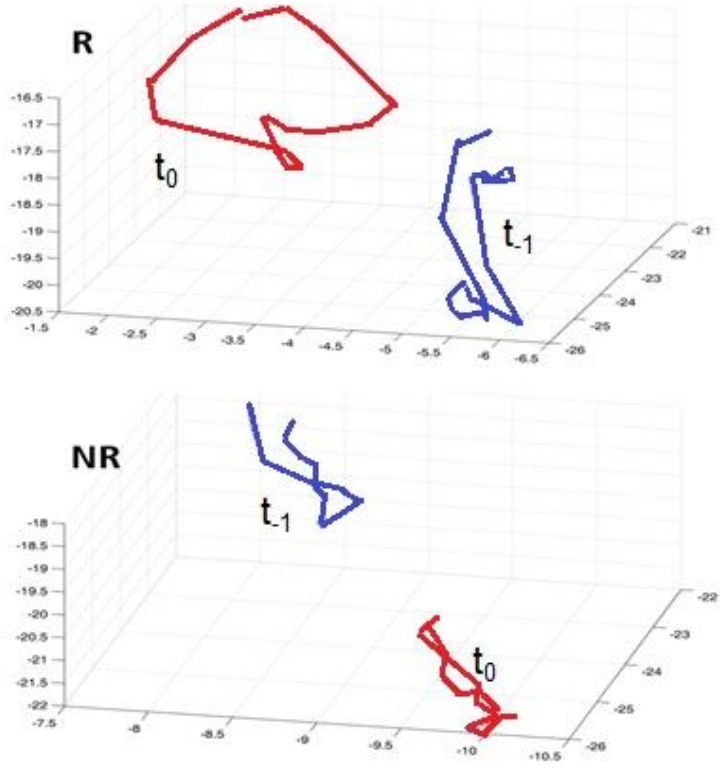

Fig. 2: Examples of 3DTJ changes in a responder (R, top panel) and in a non-responder (NR, bottom panel) patient.

Volumetric response was adjudicated by a core-lab based on echocardiographic LV end-systolic volume (LVESV) reduction at follow-up. Patients were classified as responder $(\mathrm{R})$ if they showed a LVESV reduction equal or greater than $15 \%$, non-responder (NR) if they did not.

\section{Results}

At follow-up, volumetric responders were 34/50 (68\%). Concordance between $\Delta S_{1} / S_{2}$ (with cut-off value fixed at $\left.\Delta \mathrm{S}_{1} / \mathrm{S}_{2}<0\right)$ and volumetric response was $82 \%$ overall (41/50), 91\% in R (31/34), 62\% in NR (10/16). Clinical and ECG features in our population and in $\mathrm{R}$ and NR subgroups are reported in Table 1 and Table 2, at baseline and at follow-up. At baseline, between R and NR there was no significant difference ( $p>0.05)$ apart from $\Delta \mathrm{S}_{1} / \mathrm{S}_{2}$, which demonstrated highly significant different values in the two groups. When used to predict patient outcome, it showed sensitivity of $91 \%$, specificity equal to $62 \%$, positive predictive value equal to $84 \%$ and negative predictive value equal to $77 \%$.

Non-concordant patients were older $(73 \pm 8$ years $)$, predominantly with IHD (7/9) and mostly NR (6/9), but no differences in baseline QRS duration and in LVEF were found between concordant and non-concordant subgroups (Table 3).
Table 1. Baseline clinical and ECG features in our population and in responders (R) and non-responders (NR) subgroups.

\begin{tabular}{lccc}
\hline & All & $\mathrm{R}$ & $\mathrm{NR}$ \\
\hline Patients (n, male) & $50(35)$ & $34(23)$ & $16(12)$ \\
Age (years, m \pm sd) & $69 \pm 10$ & $69 \pm 11$ & $69 \pm 11$ \\
IHD (n, \%) & $28(56)$ & $17(50)$ & $11(69)$ \\
Atrial fibrillation (n) & 7 & 4 & 3 \\
Upgrade from PM/ICD (n) & 9 & 6 & 3 \\
QRS morphology LBBB (n) & 40 & 28 & 12 \\
QRS morphology IVD (n) & 4 & 2 & 2 \\
QRS morphology RV (n) & 8 & 5 & 3 \\
\hline IHD: ischemic heart disease; PM: pacemaker; ICD: implantable \\
cardioverter defibrillator; LBBB: left bundle branch block; IVD: ischemic \\
valvular disease; RV: right ventricle.
\end{tabular}

Table 2. Baseline and follow-up ECG and echocardiographic features (mean \pm standard deviation) in all population and in responders (R) and non-responders (NR) subgroups.

\begin{tabular}{lccc}
\hline Pre-CRT Baseline & All & R & NR \\
\hline QRS duration (msec) & $162 \pm 26$ & $165 \pm 55$ & $163 \pm 35$ \\
LVEF (\%) & $32 \pm 10$ & $30 \pm 10$ & $35 \pm 10$ \\
LVESV (ml) & $75 \pm 38$ & $79 \pm 40$ & $63 \pm 28$ \\
$\Delta$ S $_{1} / \mathrm{S}_{2}$ & $3.4 \pm 68$ & $-22 \pm 27 *$ & $48 \pm 90$ \\
At Follow-up & & & \\
\hline QRS duration (msec) & $135 \pm 24$ & $131 \pm 24$ & $145 \pm 25$ \\
LVEF (\%) & $43 \pm 12$ & $45 \pm 13$ & $39 \pm 9$ \\
LVESV (ml) & $52 \pm 24$ & $45 \pm 19$ & $65 \pm 27$ \\
\hline LVEF: left ventricular ejection fraction; LVESV: left ventricular end \\
systolic volume; *p $<0.05$ R vs NR.
\end{tabular}

Table 3. Features of non-concordant (NC) and concordant (C) groups (concordance between 3DTJ metric prediction and volumetric response).

\begin{tabular}{lcc}
\hline & C & NC \\
\hline Patients (n, \%) & $41(78)$ & $9(22)$ \\
Age (years) & $68 \pm 11$ & $73 \pm 8$ \\
IHD (n, \%) & $20(49)$ & $8(89)$ \\
CRT - NR (n, \%) & $9(22)$ & $7(78)$ \\
QRS morphology LBBB (n, \%) & $31(76)$ & $9(100)$ \\
PACE RV (n, \%) & $6(15)$ & $2(22)$ \\
Pre-CRT QRS duration (msec) & $163 \pm 27$ & $159 \pm 29$ \\
Post-CRT QRS duration (msec) & $132 \pm 22$ & $131 \pm 21$ \\
Pre-CRT LVEF (\%) & $31 \pm 10$ & $31 \pm 10$ \\
Follow-up LVEF (\%) & $44 \pm 12$ & $37 \pm 12$ \\
Pre-CRT LVESV (ml) & $75 \pm 42$ & $73 \pm 33$ \\
Follow-up LVESV (ml) & $48 \pm 21$ & $64 \pm 30$ \\
\hline IHD: ischemic heart disease; LBBB: left bundle branch block; RV: \\
right ventricle; LVEF: left ventricular ejection fraction; LVESV: left \\
ventricular end systolic volume.
\end{tabular}




\section{Discussion and Conclusion}

In this perspective study the CS lead cathode pole trajectory reconstruction is used to assess the correlation between acute trajectory modifications induced by biventricular pacing in CRT and the response to CRT in a multicenter population.

Our results point at a smoother and more circular trajectory as the immediate result of the CRT pacing turning-on in the $\mathrm{R}$ group and confirm in a multicenter population the results previously obtained in the single center pilot study [7]. Therefore, these additional results, based on completely blinded predictions made months before the actual clinical response was assessed, support metrics of 3DTJ to acutely predict long-term volumetric response to CRT, although present data need confirming on the entire study population.

This analysis could be easily performed by means of fluoroscopic data recordings currently acquired during CRT implants without any incremental costs in terms of time, operator-expertise and supplementary technology. In Addition, since this investigation does not interfere with the implant procedure, the analysis of the trajectory could be explored further and compared with more established or newly proposed methods targeting ventricular dyssynchrony.

3DTJ depicts aspects of CRT delivery effects on LV mechanics. It could be speculated that the "opening" of the trajectory induced locally by the pacing in R patients (see, e.g., Figure 2, top panel), and quantified by a negative $\Delta S_{1} / S_{2}$ value in these patients, can reflect a contraction which is more coordinated/integrated with the overall ventricle movement during the different phases of the cardiac cycle. But further investigation is required to understand the mechanistic significance of 3DTJ and the derived parameters.

In conclusion, these preliminary data show that $3 \mathrm{D}$ trajectories could describe features of resynchronization start-up in CRT recipients and may help to understand the reasons of therapy failure in non-responder patients.
Importantly, its investigational use as an intraoperatory, real-time guidance for selecting LV pacing sites may open new clear and impactful translational opportunities for CRT patients' selection and therapy optimization in order to significantly improve the response rate.

\section{References}

[1] F.A. McAlister, Cardiac resynchronization therapy for patients with left ventricular systolic dysfunction: a systematic review, JAMA 2007; 297(22): 2502-2514.

[2] J.G.F. Cleland, The effect of cardiac resynchronization on morbidity and mortality in heart failure, The New England Journal of Medicine 2005;352:1539-1549.

[3] W. T. Abraham, Cardiac resynchronization in chronic heart failure, The New England Journal of Medicine 2002;346:1845-1853.

[4] C.M. Yu et al, LV reverse remodeling but not clinical improvement predicts long-term survival after cardiac resynchronization therapy. Circulation 2005;112:15801586.

[5] J. Gorscan, Echocardiographic assessment of ventricular dyssynchrony. Current Cardiology Reports 2008;10(3):211217.

[6] C. Corsi, C. Tomasi, D. Turco, M. Margheri, C. Lamberti, S. Severi, 3D dynamic position assessment of the coronary sinus lead in cardiac resynchronization therapy, Medical and Biological Engineering and Computing 2011;49:901-908.

[7] C. Tomasi, C. Corsi, D. Turco, S. Severi. An exploratory study on coronary sinus lead tip trajectory changes in cardiac resynchronization therapy, Heart Rhythm, 2013;10(9):13601367.

Address for correspondence.

Cristiana Corsi

DEI, University of Bologna, Campus di Cesena

Via dell'Università 50, 47521 Cesena, Italy

E-mail address: cristiana.corsi3@unibo.it 